\title{
Serum Uric Acid: A Murderer or Bystander for Cardiac-related Mortality?
}

\author{
Cheng-Wei Liu'1(D)
}

In this issue of The Journal of Rheumatology, Colantonio, et al ${ }^{1}$ conducted a case-cohort study from the REGARDS study to evaluate whether the association between serum uric acid (SUA) and sudden cardiac death, and between SUA and incident coronary heart disease (CHD) events, is confounded by SLC2A9 single-nucleotide polymorphisms (SNPs). Incident CHD events were the composites of nonfatal myocardial infarction (MI) or CHD deaths. The final analyzable data consisted of 840 patients with 235 sudden cardiac death and 851 incident CHD events. These patients were divided into the 3 groups by SUA tertiles. SUA was independently associated with sudden cardiac death but not with CHD events after the authors adjusted for SC2A9 SNPs on the top of baseline characteristics (age, sex, race, BMI, socioeconomic status, lifestyle choice, medical history), laboratory data (lipid profiles and C-reactive protein [CRP]), electrocardiographic left ventricular hypertrophy (LVH), and use of medication (statin, antihypertensive medication, diuretics, and allopurinol). Notably, some of the confounders were also the consequences of elevated SUA in the study. ${ }^{1}$ Previous studies have shown that SUA was associated with increased CRP, ${ }^{2,3}$ metabolic syndrome, ${ }^{4}$ hypertension, ${ }^{5,6} \mathrm{LVH},{ }^{4,7}$ chronic kidney disease (CKD), ${ }^{8}$ and atrial fibrillation. ${ }^{9}$ In other words, the pathological effects of elevated SUA on CHD events were eliminated when these confounders were adjusted. Additionally, the REGARDS study excluded patients with medical histories of CHD at baseline, but baseline silent myocardial ischemia should not be ignored in the study period. A recent study used autopsy information and found that a substantial number of individuals had sudden cardiac death without a prior diagnosis of coronary

${ }^{I}$ C.W. Liu, MD, Division of Cardiology, Department of Internal Medicine, Tri-Service General Hospital Songshan Branch, and Graduate Institute of Clinical Medicine, College of Medicine, National Taiwan University, Taipei, Taiwan.

The author declares no conflicts of interest relevant to this article.

Address correspondence to Dr. C.W. Liu, Division of Cardiology, Department of Internal Medicine, Tri-Service General Hospital Songshan Branch, National Defense Medical Center, Taipei, Taiwan 10581, No. 131, Jiankang Rd., Songshan Dist., Taipei City 105, Taiwan (ROC).

Email:issac700319@gmail.com. artery disease (CAD) and concluded that "many individuals who experienced sudden cardiac death associated with CAD had a previously undetected MI at autopsy." ${ }^{10}$ Therefore, the insignificant association between SUA and CHD events should be interpreted more carefully in the study by Colantonio, et al. ${ }^{1}$ Besides, another systematic review and metaanalysis showed the significant association that "hyperuricemia may increase the risk of CHD events." 11

It is essential to understand the SUA basal mechanism for cardiovascular diseases (CVD) as we explore the field between SUA and CVD. SUA is a weak acid, with an ionization constant of acid (acid dissociation constant [pKa]) of 5.75-10.3. At the physiological pH of 7.40 of the extracellular compartment, $99 \%$ of uric acid is in its ionized form as monosodium urate in the blood. ${ }^{12}$ Although soluble SUA and urate crystals are interchangeable in some conditions, they are not always synonymous clinically. In discussions about the association between SUA and CVD, the term $S U A$ is most used in published articles and in the REGARDS study. ${ }^{13}$ Soluble SUA is clinically measured differently than urate crystals, which is considered a harmful deposition in organs or tissues. Therefore, the term urate-lowering therapy (ULT) is better used to illustrate the lowering of detrimental levels of deposition in humans, although a few studies used the term uric acid-lowering therapy. The therapeutic effects are not only limited to lowering SUA but also to inhibiting the inflammatory response and reactive oxidative stress through xanthine oxidase inhibitor, a kind of ULT. ${ }^{14}$

SUA pathologically induces vascular inflammation and reactive oxidative stress, thereby activating the renin-angiotensinaldosterone system. ${ }^{15}$ These mechanisms may partially explain the association between elevated SUA and CVD, independent of urate deposition in kidneys. Urate deposition starts in joint spaces and kidneys as the SUA level exceeds the physiological concentration of $6.8 \mathrm{mg} / \mathrm{dL} .{ }^{12}$ In clinical practice, hyperuricemia is generally defined as an SUA $\geq 7 \mathrm{mg} / \mathrm{dL}$ in men or $\geq 6 \mathrm{mg} / \mathrm{dL}$ in women. However, various thresholds of SUA are used for different races and ethnicities. ${ }^{4}$ The thresholds of elevated SUA for different races and ethnicities are hard to use

\section{See Serum urate and sudden death, page 1745}


clinically and they lack external validation, especially the dichotomies, tertiles, or quartiles of SUA levels used in various studies. These cut-off values cannot be applied clinically. Therefore, the well-acknowledged definition of hyperuricemia may be used as a sensitivity analysis in the study by Colantonio, et al, and it helps investigators pool study data for further statistical analysis. For statistical analyses in future studies, both definitions of elevated SUA - the universal definition of hyperuricemia and SUA thresholds for different races and ethnicities-should be used.

Colantonio, et al ${ }^{1}$ conclude that $S L C 2 A 9$ are not confounders in the association between serum urate levels and sudden cardiac death, but SLC2A9 and glucose transporter 9 (GLUT-9) may be behind higher SUA concentrations, which are associated with sudden cardiac death. However, the study by Colantonio, et al is an association study, and the hypothesis cannot be established directly without a plausibility study. The formation of SUA is not only from the fructose pathway but also primarily involves purine metabolism. During this process, oxidation and free radicals may induce inflammation and atherosclerosis. In addition, oxidative stress is also postulated to act on ryanodine receptors and inhibit nitric oxide formation. ${ }^{16}$ It results in cardiac relaxation abnormality in experimental models ${ }^{17}$; in humans, this is known as left ventricular diastolic dysfunction. ${ }^{3,18}$ Elevated SUA induces nitric oxide deficiency in endothelial cells, inflammatory response in pancreatic islet cells, and interstitial fibrosis in kidneys. ${ }^{15,19}$ SUA is a terminal product of purine metabolism; a critical enzyme essential for metabolizing xanthine and hypoxanthine into SUA is xanthine oxidase, generating reactive oxygen species and inflammatory responses. ${ }^{15}$ These mechanisms may lead to sudden cardiac death or incident CHD events. Interactions exist between SUA and cardiovascular risk factors such as obesity, diabetes mellitus, ${ }^{20} \mathrm{CKD},{ }^{8}$ and severity of underlying diseases. ${ }^{21}$ Intake of fructose results in elevated SUA levels, especially in individuals with diabetes and obesity; facilitative GLUT-9, as both fructose and uric acid transporter, may explain the association between SUA and cardiometabolic abnormalities. ${ }^{20}$ Colantonio, et al $l^{1}$ showed that SUA was associated with sudden cardiac death independent of the $S L C 2 A 9$ gene but was not independently associated with CHD events; this is not good enough to explore a causal effect among SUA, SLC2A9, and GLUT-9 by a cohort study. A conditional gene knockout mice model may be conducted to investigate whether the effects of SUA on sudden cardiac death is modified by the presence of the SLC2A9 gene and through GLUT-9.22

\section{REFERENCES}

1. Colantonio LD, Reynolds RJ, Merriman TR, Gaffo A, Singh JA, Plante TB, et al. Higher serum urate levels are associated with an increased risk for sudden cardiac death. J Rheumatol 2021; 48:1745-53.

2. Spiga R, Marini MA, Mancuso E, Di Fatta C, Fuoco A, Perticone $\mathrm{F}$, et al. Uric acid is associated with inflammatory biomarkers and induces inflammation via activating the NF- $\kappa \mathrm{B}$ signaling pathway in HepG2 cells. Arterioscler Thromb Vasc Biol 2017;37:1241-9.

3. Liu CW, Chen JH, Tseng GS, Chen KH, Hwang JJ, Yang WS, et al. Association between low-grade inflammation and left ventricular diastolic dysfunction in patients with metabolic syndrome and hyperuricemia. Acta Cardiol Sinica 2020;36:483-92.
4. Liu CW, Chen KH, Tseng CK, Chang WC, Wu YW, Hwang JJ. The dose-response effects of uric acid on the prevalence of metabolic syndrome and electrocardiographic left ventricular hypertrophy in healthy individuals. Nutr Metab Cardiovasc Dis 2019;29:30-8.

5. Liu CW, Ke SR, Tseng GS, Wu YW, Hwang JJ. Elevated serum uric acid is associated with incident hypertension in the health according to various contemporary blood pressure guidelines. Nutr Metab Cardiovasc Dis 2021;31:1209-18.

6. Gill D, Cameron AC, Burgess S, Li X, Doherty DJ, Karhunen V, et al. Urate, blood pressure, and cardiovascular disease. Hypertension 2021;77:383-92.

7. Liu C, Chang W, Pan R. P282 Elevated serum uric acid associated with both electrocardiographic and echocardiographic left ventricular hypertrophy independent of blood pressure in healthy individuals [abstract]. European Heart J 2020;41 Suppl 1:ehz872.102.

8. Luo Q, Xia X, Li B, Lin Z, Yu X, Huang F. Serum uric acid and cardiovascular mortality in chronic kidney disease: a meta-analysis. BMC Nephrology 2019;20:18.

9. Tamariz L, Hernandez F, Bush A, Palacio A, Hare JM. Association between serum uric acid and atrial fibrillation: a systematic review and meta-analysis. Heart Rhythm 2014;11:1102-8.

10. Vähätalo JH, Huikuri HV, Holmström LTA, Kenttä TV, Haukilahti MAE, Pakanen L, et al. Association of silent myocardial infarction and sudden cardiac death. JAMA Cardiol 2019;4:796-802.

11. Li M, Hu X, Fan Y, Li K, Zhang X, Hou W, et al. Hyperuricemia and the risk for coronary heart disease morbidity and mortality a systematic review and dose-response meta-analysis. Sci Rep 2016;6:19520

12. Grassi D, Ferri L, Desideri G, Di Giosia P, Cheli P, Del Pinto R, et al. Chronic hyperuricemia, uric acid deposit and cardiovascular risk. Curr Pharm Des 2013;19:2432-8.

13. Chaudhary NS, Bridges SL Jr, Saag KG, Rahn EJ, Curtis JR, Gaffo A, et al. Severity of hypertension mediates the association of hyperuricemia with stroke in the REGARDS case cohort study. Hypertension 2020;75:246-56.

14. Liu CW, Chang WC, Lee CC, Shau WY, Hsu FS, Wang ML, et al. The net clinical benefits of febuxostat versus allopurinol in patients with gout or asymptomatic hyperuricemia-a systematic review and meta-analysis. Nutr Metab Cardiovasc Dis 2019;29:1011-22.

15. Feig DI, Kang DH, Johnson RJ. Uric acid and cardiovascular risk. N Engl J Med 2008;359:1811-21.

16. Tziomalos K, Hare JM. Role of xanthine oxidoreductase in cardiac nitroso-redox imbalance. Front Biosci 2009;14:237-62.

17. Jia G, Habibi J, Bostick BP, Ma L, DeMarco VG, Aroor AR, et al. Uric acid promotes left ventricular diastolic dysfunction in mice fed a Western diet. Hypertension 2015;65:531-9.

18. Tu CM, Tseng GS, Liu CW. Serum uric acid may be associated with left ventricular diastolic dysfunction in military individuals. Mil Med 2020 Oct 30; usaa413.

19. Joosten LAB, Crişan TO, Bjornstad P, Johnson RJ. Asymptomatic hyperuricaemia: a silent activator of the innate immune system. Nat Rev Rheumatol 2020;16:75-86.

20. Doblado M, Moley KH. Facilitative glucose transporter 9, a unique hexose and urate transporter. Am J Physiol Endocrinol Metab 2009;297:E831-5.

21. Liu CW, Liao PC, Chen KC, Chiu YW, Liu YH, Ke SR, et al. Relationship of serum uric acid and Killip class on mortality after acute ST-segment elevation myocardial infarction and primary percutaneous coronary intervention. Int J Cardiol 2017;226:26-33.

22. Merriman TR. An update on the genetic architecture of hyperuricemia and gout. Arthritis Res Ther 2015;17:98. 